\title{
RADIAL STRUCTURE IN THE TW Hya CIRCUMSTELLAR DISK
}

\author{
R. L. Akeson ${ }^{1}$, R. Millan-Gabet ${ }^{1}$, D. R. Ciardi ${ }^{1}$, A. F. Boden ${ }^{2}$, A. I. Sargent ${ }^{2}$, J. D. Monnier ${ }^{3}$, H. McAlister ${ }^{4}$, \\ T. Ten BrummelaAR ${ }^{4}$, J. SturmanN ${ }^{4}$, L. Sturmann ${ }^{4}$, and N. Turner ${ }^{4}$ \\ ${ }^{1}$ NASA Exoplanet Science Institute, Caltech, Pasadena, CA 91125, USA \\ ${ }^{2}$ Department of Astronomy, Caltech, Pasadena, CA 91125, USA \\ ${ }^{3}$ Department of Astronomy, University of Michigan, 830 Dennison Building, 500 Church Street, Ann Arbor, MI 48109, USA \\ ${ }^{4}$ Center for High Angular Resolution Astronomy, Georgia State University, Atlanta, GA 30302, USA \\ Received 2010 September 16; accepted 2010 December 14; published 2011 January 25
}

\begin{abstract}
We present new near-infrared interferometric data from the CHARA array and the Keck Interferometer on the circumstellar disk of the young star, TW Hya, a proposed "transition disk." We use these data, as well as previously published, spatially resolved data at $10 \mu \mathrm{m}$ and $7 \mathrm{~mm}$, to constrain disk models based on a standard flared disk structure. We find that we can match the interferometry data sets and the overall spectral energy distribution with a three-component model, which combines elements at spatial scales proposed by previous studies: optically thin, emission nearest the star, an inner optically thick ring of emission at roughly $0.5 \mathrm{AU}$ followed by an opacity gap and, finally, an outer optically thick disk starting at $\sim 4$ AU. The model demonstrates that the constraints imposed by the spatially resolved data can be met with a physically plausible disk but this requires a disk containing not only an inner gap in the optically thick disk as previously suggested, but also a gap between the inner and outer optically thick disks. Our model is consistent with the suggestion by Calvet et al. of a planet with an orbital radius of a few AU. We discuss the implications of an opacity gap within the optically thick disk.
\end{abstract}

Key words: circumstellar matter - stars: individual (TW Hya)

\section{INTRODUCTION}

A detailed understanding of the timescales and physical triggers in the dissipation of primordial circumstellar disks is a key input to understanding the process of planet formation in these disks. Early studies of the spectral energy distributions (SEDs) of young stars identified a subset of the disk population that had a small (or no) near-IR excess and a substantial midto far-IR excess (see, e.g., Strom et al. 1989). Such sources are now generally referred to as transition disks and are roughly defined as having an opacity deficit in the inner disk with an optically thick outer disk. Several physical processes have been proposed for the formation mechanism of the opacity hole in transition disks including grain growth, photoevaporation, planet formation, and stellar multiplicity. These processes have different implications for other disk properties such as the accretion rate; see Najita et al. (2007) for a detailed discussion. Recent surveys, particularly with Spitzer, have greatly expanded the number of proposed transition disks (e.g., Furlan et al. 2009; Muzerolle et al. 2010; Merín et al. 2010). High angular resolution observations have shown that at least some of these inner holes are due to stellar binarity (e.g., CoKu Tau 4; Ireland \& Kraus 2008), but the physical mechanism at work in the majority of transitional disks has yet to be clearly determined.

One of the nearest transition disks is TW Hya, located at $\sim 50 \mathrm{pc}$ in the cluster of the same name. Despite an estimated age of 8-10 million years (Hoff et al. 1998; Webb et al. 1999), TW Hya shows many signatures of an actively accreting T Tauri star, including an $\mathrm{H} \alpha$ emission line width of $220 \AA$ (Webb et al. 1999). TW Hya's circumstellar disk is particularly well studied. Analysis of the SED by Calvet et al. (2002) led them to conclude that the disk is optically thin inside of $4 \mathrm{AU}$ and they proposed that this gap may be due to a giant planet within the disk.
The TW Hya disk has been spatially resolved at many wavelengths. Wilner et al. (2000, 2003), Qi et al. (2004), and Isella et al. (2009) resolved the outer disk in the millimeter regime and found range of $70-140 \mathrm{AU}$ for the outer radius of the dust emission. Eisner et al. (2006) measured a single visibility point in the $K$ band with the Keck Interferometer (KI) and found resolved emission from which they inferred an inner radius of $0.06 \mathrm{AU}$ for a population of optically thin dust and the combination of the KI data with the SED implied sub-micron sized grains. Higher resolution millimeter imaging at $7 \mathrm{~mm}$ with the Very Large Array (VLA) by Hughes et al. (2007) confirmed the inner radial size of $\sim 4$ AU derived by Calvet et al. (2002) from the SED. However, $10 \mu \mathrm{m}$ interferometry observations by Ratzka et al. (2007) with VLTI using the MIDI instrument were consistent with a disk transitioning to optically thick at a radius of 0.8 AU. Recently, the inner disk of TW Hya came under even more scrutiny with the claim of a radial velocity detected planet with a semi-major axis of 0.04 AU (Setiawan et al. 2008), although an alternative explanation is that the radial velocity variations are due to a cool stellar spot (Huélamo et al. 2008). Additional observations of near-IR radial velocity variations with the same period but a lower amplitude than in the optical have been detected (Figueira et al. 2010), as expected for a spot.

Spatially resolved observations are crucial in constraining the physical structure of the inner disk of TW Hya and distinguishing which mechanisms are involved in clearing the inner disk. Here, we present new near-IR interferometric observations with a range of baseline lengths from Center for High Angular Resolution Astronomy (CHARA) and KI, which directly probe the inner few AU. These observations are then combined with existing data to constrain circumstellar disk models. The observations are described in Section 2, the circumstellar disk models in Section 3, the implications discussed in Section 4, and the conclusions given in Section 5. 
Table 1

Calibrators and Angular Sizes for KI and CHARA Observations

\begin{tabular}{lcc}
\hline \hline Calibrator & Angular Diameter (mas) & Instrument \\
\hline HD 99934 & $0.30 \pm 0.04$ & KI \\
HD 97940 & $0.16 \pm 0.03$ & KI \\
HD 97023 & $0.18 \pm 0.02$ & KI \\
HD 94034 & $0.14 \pm 0.01$ & KI, CHARA \\
HD 98048 & $0.15 \pm 0.01$ & KI, CHARA \\
\hline
\end{tabular}

\section{OBSERVATIONS AND DATA ANALYSIS}

\subsection{CHARA Observations}

Observations were taken at the CHARA array, which is a long-baseline, six-element interferometer operated by Georgia State University with direct detection instruments working at optical to near-IR wavelengths (ten Brummelaar et al. 2005). These data were collected at $K$ band (central wavelength = $2.13 \mu \mathrm{m}$ ) with the CHARA Classic instrument, a fringe scanning, single baseline combiner. Two baselines were chosen to bracket the Eisner et al. (2006) data point; we used the S1-S2 and $\mathrm{S} 1-\mathrm{W} 1$ baselines. The calibrators and angular sizes are given in Table 1. As the calibrators are small compared to the angular resolution, the uncertainties in the angular size of the calibrators do not contribute substantially to the final measurement uncertainties.

Data reduction and calibration were performed using the standard CHARA pipeline (ten Brummelaar et al. 2005). The calibrated data, including the projected baseline lengths, are given in Table 2. As is apparent from the relatively large error bars in Table 2, the combination of relatively faint $V$ - and $K$-band magnitudes and low elevation $\left(<30^{\circ}\right)$ made these observations challenging for CHARA. However, a parallel analysis using an independent pipeline employing somewhat different methods developed by one of us (J.D.M.) yielded consistent results, indicating that the results are robust in spite of those difficulties.

\subsection{Keck Interferometer Observations}

A single observation of TW Hya was made with the KI on 2009 May 14 at the $K$ band $(2.18 \mu \mathrm{m})$, followed by observations of calibrators HD 94034 and HD 98048. These observations were reduced using the standard KI data calibration method (Colavita et al. 2003), including calibration for the measured flux bias. ${ }^{5}$ The calibrated normalized squared visibility is $0.97 \pm 0.03$ (Table 2). Additionally, we re-calibrated the $K$-band data point from Eisner et al. (2006) to account for the flux bias and found a squared visibility of $0.92 \pm 0.046$.

\subsection{Geometric Size Estimates}

As described above, previous studies have resolved the circumstellar disk around TW Hya at wavelengths from the mid-IR to the millimeter. In particular, Ratzka et al. (2007) and Hughes et al. (2007) used the VLTI and the VLA, respectively, and derived substantially different values for the inner radius of the optically thick dust disk. We include the spatially resolved measurements of both groups in our modeling in an attempt to find a disk structure consistent with both these data sets, as well as with our near-IR data. To guide the range of models considered, we first made geometric size estimates for both our data and the VLTI and VLA data sets. These are not meant

\footnotetext{
5 http://nexsci.caltech.edu/software/KISupport/dataMemos/fluxbias.pdf
}

Table 2

Calibrated Squared Visibility Measurements from KI and CHARA

\begin{tabular}{lcccc}
\hline \hline Instrument & MJD & Proj. Baseline $(\mathrm{m})$ & Vis $^{2}$ & $\sigma$ \\
\hline KI & 53481.323 & 61.7 & 0.92 & 0.046 \\
KI & 54965.246 & 65.0 & 0.97 & 0.030 \\
CHARA & 54927.223 & 12.6 & 0.823 & 0.079 \\
CHARA & 54927.239 & 13.4 & 0.957 & 0.066 \\
CHARA & 54927.254 & 14.4 & 0.872 & 0.060 \\
CHARA & 54927.269 & 15.6 & 0.808 & 0.065 \\
CHARA & 54927.287 & 17.1 & 0.979 & 0.098 \\
CHARA & 54928.265 & 206.9 & 0.877 & 0.136 \\
\hline
\end{tabular}

to correspond to specific physical models but to provide size estimates that indicate which parts of the disk contribute most to which data sets.

In the near-IR, we jointly fit the data from KI and CHARA with a Gaussian and with a completely incoherent flux component (flux on scales much larger than the fringe spacing will contribute incoherently). The relative beam sizes for KI (50 mas; 2.7 AU diameter) and CHARA (1.2 arcsec; 64.4 AU diameter) were properly accounted for. One of the crucial input parameters for the near-IR fits is the fractional flux from the disk at $K$ band. This can be derived several ways, including SED fitting and veiling estimates. Near-IR veiling measurements have been made by Johns-Krull \& Valenti (2001) and Eisner et al. (2010), and are consistent with each other. Here we use the veiling measurement of Johns-Krull \& Valenti (2001) of $r_{k}=0.07 \pm 0.04$, where $r_{k}$ is the ratio of the non-stellar flux to the stellar flux. This estimate is more precise than can be obtained by SED fitting unless a complete and contemporaneous set of photometry is obtained in the optical and near-IR, which we do not have. Any change in the veiling will directly impact the derived size of the near-IR emission, with a higher veiling value corresponding to a larger fractional incoherent excess or larger spatial scale for the excess emission. We note that a veiling value $1 \sigma$ lower than that measured by Johns-Krull \& Valenti (2001; i.e., $\left.r_{k}=0.03\right)$ would rule out the resolved visibility arising only from an incoherent contribution as it would require a negative flux to match the observed visibility. The best-fit Gaussian has a radius of $1.3_{-1.2}^{+1.8}$ mas $\left(0.07_{-0.06}^{+0.1} \mathrm{AU}\right)$ with a $\chi^{2}=2.7$ while a incoherent contribution is a statistically better fit with a fractional flux excess of $0.032 \pm 0.027$ and a $\chi^{2}=1.2$ (Figure 1).

Although the large uncertainties of the CHARA data, particularly the long baseline point, limit the spatial constraints which can be set on the near-IR emission size, we can use these data to constrain the general distribution of the emission. Averaging the short baseline CHARA points gives a normalized squared visibility of $0.88 \pm 0.03$, which shows that the near-IR flux contains resolved emission on even a $\sim 15 \mathrm{~m}$ baseline. This is inconsistent (at the $4 \sigma$ level) with the near-IR emission predominantly arising from a thin ring of material at the dust sublimation radius $(0.02 \mathrm{AU}$ ) or at the $0.06 \mathrm{AU}$ radius derived by Eisner et al. (2006) from KI data alone, as both these scales would produce a visibility of 1.0 on this short baseline. As thermal emission from dust at $2 \mu \mathrm{m}$ is strongly temperature dependent, and will therefore decrease with radius, this suggests a significant scattering component in the CHARA FOV (radii $<32 \mathrm{AU}$ ) as has been observed at larger radii.

For the VLTI fits, we used the visibility measurements of Ratzka et al. (2007) and subtracted the stellar component derived in Section 3.1. The fractional excess calculated ranges from 0.8 to 0.95 across the $N$ band. Figure 2 shows the VLTI visibilities, derived fractional excess, and the fit ring radius 

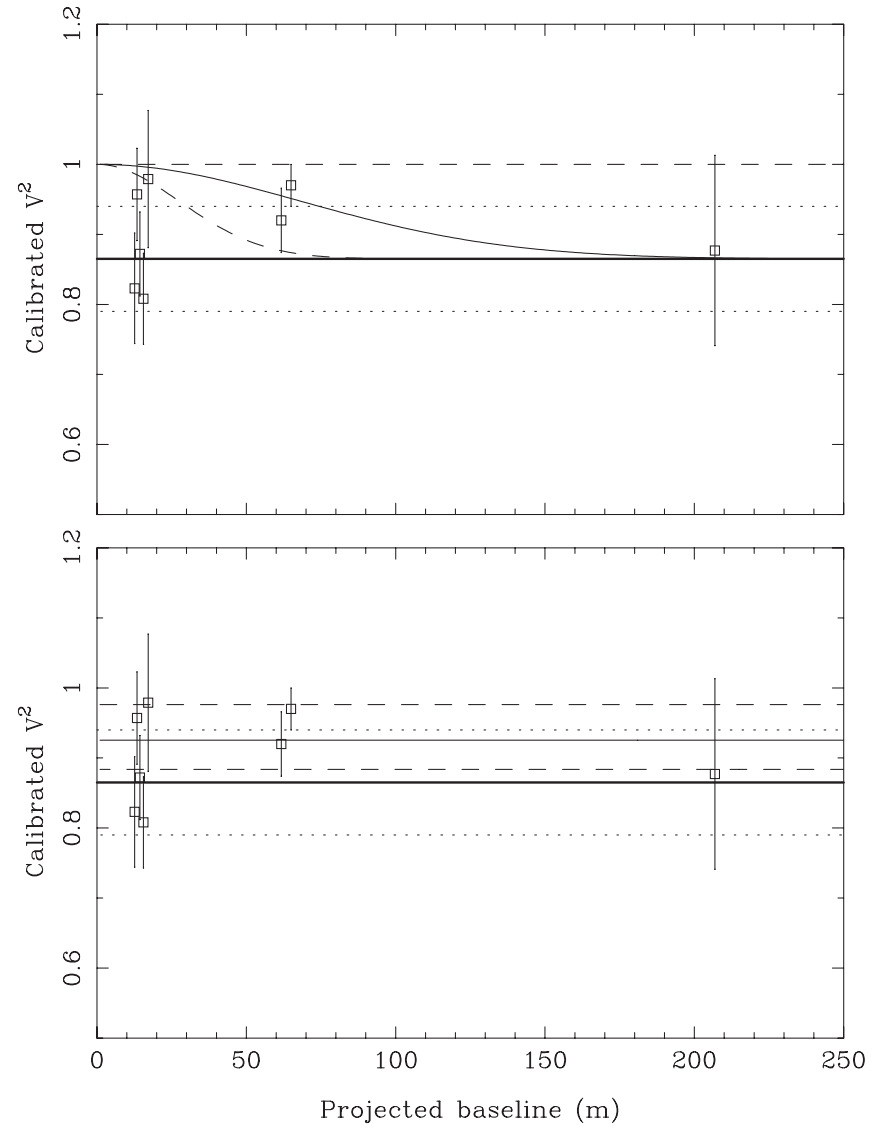

Figure 1. Best-fit Gaussian (top panel, solid line) and incoherent flux (bottom panel, solid line) fit to the KI and CHARA data. The $1 \sigma$ uncertainties in the fit are shown as dashed lines. The stellar contribution (in visibility squared, bold line) and uncertainty (dotted lines) are also shown.

at each wavelength. The ring radius as a function of wavelength does not increase with increasing wavelength, as would arise from sampling a flared disk with a radially decreasing temperature, but instead, remains relatively constant from 10 to $13 \mu \mathrm{m}$. As discussed by Ratzka et al. (2007), this demonstrates that the mid-IR flux is dominated by emission from a localized region, such as a vertically extended inner disk rim. The VLTI data were binned by wavelength in our model fitting to minimize the calculation times.

As discussed by Hughes et al. (2007), the VLA 7 mm visibility amplitude as a function of $u v$ distance passes through a null (see Figure 2 in Hughes et al. 2007) requiring a distinct structure at that spatial frequency rather than continuous emission. The null in the visibility corresponds to a thin ring at $\sim 4 \mathrm{AU}$ (Hughes et al. 2007).

\section{CIRCUMSTELLAR DISK MODELS}

In this section, we present models for the new and previously existing data sets on the TW Hya disk. For the stellar parameters, we use the values from Webb et al. (1999) of $T_{\text {eff }}=4000 \mathrm{~K}$ and $R_{*}=1.0 R_{\odot}$. A distance of $53.7 \pm 6.2 \mathrm{pc}$ from analysis of re-reduced Hipparcos data by van Leeuwen (2007) was used. Scattered light and resolved millimeter emission imaging reveal that the disk is nearly face-on, with estimates ranging from $7^{\circ}(\mathrm{Qi}$ et al. 2004) to $12^{\circ}$ (Isella et al. 2009) in the millimeter and $<4^{\circ}$ (Weinberger et al. 2002) in scattered light. For computational convenience, we assume a completely face-on disk, i.e., $0^{\circ}$ inclination, but this has a relatively small effect on the derived
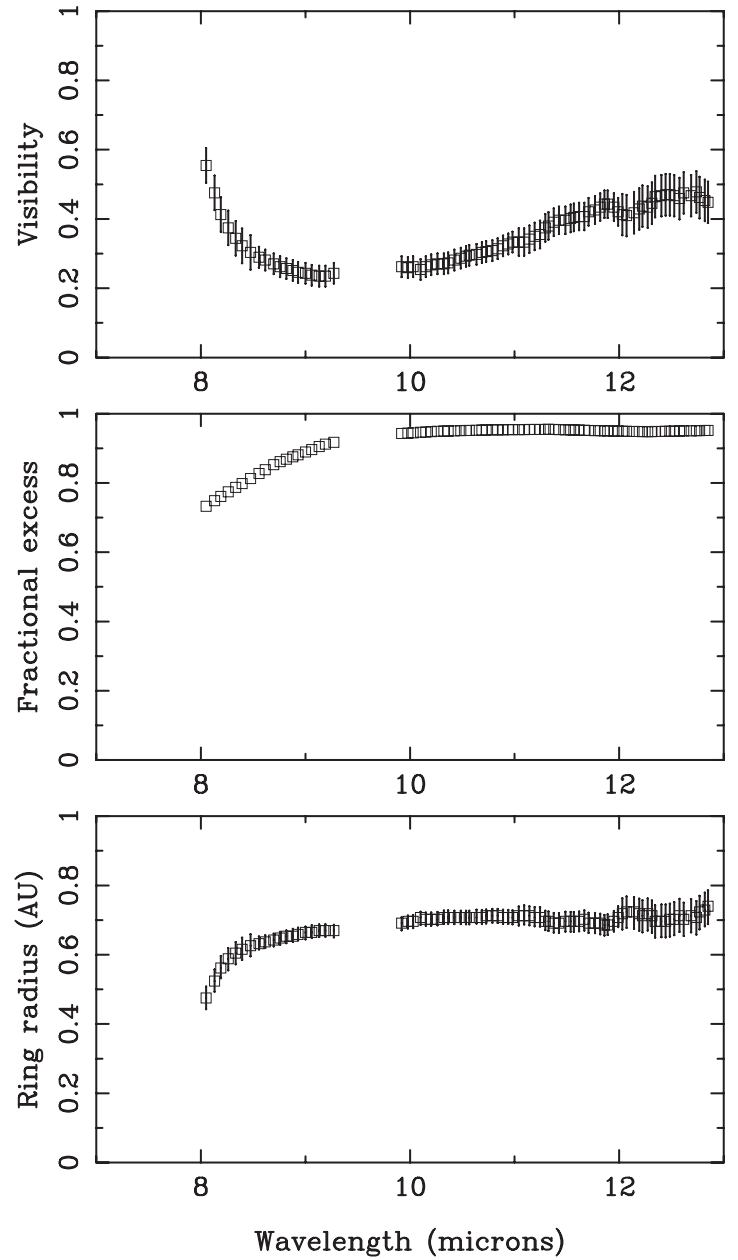

Figure 2. Visibility, fractional excess, and fit ring radius as a function of wavelength for the Ratzka et al. (2007) VLTI data excluding the silicate emission feature.

model parameters such as disk density. Based on analysis of $\mathrm{H} \mathrm{I}$ absorption in the Ly $\alpha$ emission line, Herczeg et al. (2004) find negligible extinction to TW Hya and so we use $A_{V}=0$.

\subsection{Additional Input Data and Constraints}

In addition to the three resolved data sets (near-IR, mid-IR, and millimeter) we also fit the disk SED. As TW Hya is known to be optically variable (see the discussion in Rucinski et al. 2008), the $B, V, R$, and $I$ photometry used for fitting the stellar component was derived by averaging the values in Rucinski \& Krautter (1983). These averages were fit to a $4000 \mathrm{~K} \mathrm{Kurucz}$ stellar photosphere model. The resulting fit and excess emission SED are shown in Figure 3. A 10\% uncertainty was assumed in the level of the stellar model due to the optical variability of the source. This uncertainty was propagated through the calculation of the stellar contribution at each wavelength and combined with the measurement uncertainties to produce the final uncertainties in the excess emission. We also set a minimum uncertainty of $10 \%$ in the absolute flux determination for the millimeter and submillimeter data. For fitting the disk emission, we use the derived excess emission from $3 \mu \mathrm{m}$ to $3.5 \mathrm{~cm}$. As we are concentrating here on the structure of the inner disk ( $<10 \mathrm{AU})$, we use the results of Qi et al. (2004) and Isella et al. (2009), which resolved the outer disk and applied a prior constraint on the dust disk outer size of 70-140 AU. We note that scattered light (e.g., Weinberger et al. 2002) and molecular line emission 


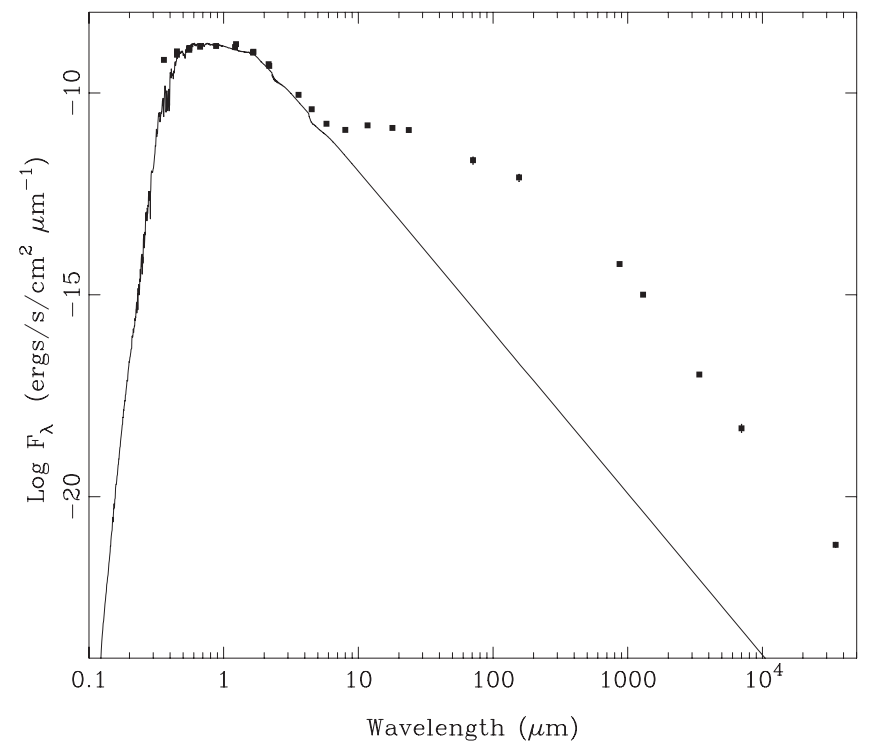

Figure 3. SED for TW Hya showing the observed photometry (points) and the fit stellar model (line). The photometry is taken from the following references: optical: Rucinski \& Krautter (1983); near-IR: Two Micron All-Sky Survey and Webb et al. (1999); IR: Spitzer IRAC and MIPS, Hartmann et al. (2005), Low et al. (2005), and Weinberger et al. (2002); (sub)millimeter and radio: Wilner et al. (2000, 2003, 2005) and Qi et al. (2004). The error bars are smaller than the point size in this plot.

(Qi et al. 2004) have been detected at much larger radii; however, in the data sets we are considering here, the dust emission is the dominant component and so we use the constraints derived from the long-wavelength continuum observations.

In this work, we are primarily concerned with the radial structure of the TW Hya disk and neglect the spectra features which constrain the composition and grain size of the dust. These features are discussed by Ratzka et al. (2007) and Najita et al. (2010).

\subsection{Modeling Approach}

For the disk models presented below, the fitting approach is to generate a large grid of models spanning a range of values for each of the model parameters. Each model is compared individually to each data set (SED, near-IR visibilities, midIR visibilities, and millimeter visibilities) and a probability that the data match that model is calculated for each data set using Bayesian formalism. The probabilities for both the individual data sets and the whole data set are accumulated over each parameter value, for all combinations of the other model parameters. This approach allows us to calculate the range of likely parameter values, rather than just a single best-fit model, as well as examine the dependence of the individual data sets on the different model parameters. We also try to use the least number of parameters and the simplest possible disk models to match the data.

\subsection{Flared Disk Model}

Following previous work on the TW Hya disk (e.g., Calvet et al. 2002; D'Alessio et al. 2001), we use a flared disk model as the underlying physical structure of the disk. We parameterized the Chiang \& Goldreich (1997, hereafter CG97) flared disk which is comprised of an interior and a superheated surface. The temperature profile for each disk component from CG97 is scaled for the stellar radius of TW Hya $\left(1.0 R_{\odot}\right)$. The dust temperatures as a function of radius $(r)$ in $\mathrm{AU}$, for the surface and interior of the disk, are then

$$
\begin{gathered}
T_{\text {surface }}=341 r^{-2 / 5} \mathrm{~K} \\
T_{\text {interior }}=93 r^{-3 / 7} \mathrm{~K} ; \text { for } r<35 \mathrm{AU} \\
T_{\text {interior }}=21 \mathrm{~K} ; \text { for } r>35 \mathrm{AU} .
\end{gathered}
$$

We adopt the CG97 radial density exponent of -1.5 , leaving the basic model with the following parameters: $\Sigma_{0}$ (surface density at $1 \mathrm{AU})$, inner radius, disk radial extent, and dust emissivity as a function of wavelength.

As we are considering only broadband data (the silicate line in the mid-IR region has been excluded from the VLTI data we are fitting) we use a simple power-law coefficient for the mass opacity

$$
\kappa_{\lambda}=\kappa_{0}\left(\frac{\lambda}{\lambda_{0}}\right)^{-\beta},
$$

where $\kappa_{o}$ is taken from CG97 and scaled to a value of $22 \mathrm{~cm}^{2}$ $\mathrm{g}^{-1}$ at $10 \mu \mathrm{m}$. In this formulation, small values of $\beta$ correspond to larger grains. Here, we use the same value for $\beta$ in both the surface layer and the interior, thus neglecting any effects due to dust settling between the surface and the interior if grain growth has occurred.

The disk flux as a function of radius is then

$$
F(r)=\frac{2 \pi}{D^{2}} B_{v}\left(1-e^{-\tau_{v}}\right) r d r
$$

where $D$ is the distance and $B_{v}$ is the Planck function. $\tau$, the disk opacity, is taken from CG97 as

$$
\begin{gathered}
\tau_{\text {interior }}=\Sigma_{0} r^{-1.5} \kappa_{\lambda} \\
\tau_{\text {surface }}=0.4 \frac{R_{\star}}{r}\left(\frac{\lambda}{1 \mu \mathrm{m}}\right)^{-\beta},
\end{gathered}
$$

where $\Sigma_{0}$ is the surface density at $1 \mathrm{AU}, R_{\star}$ is the stellar radii, and $r$ is given in AU.

The CG97 flared disk model does not explicitly include a vertical extension of the inner wall due to direct stellar irradiation. Many groups have used self-consistent physical models to examine the structure of the inner wall (see, e.g., Natta et al. 2001; Dullemond et al. 2001). The flux emitted from the rim region is highly dependent on both the inclination angle and the rim shape. As discussed by Isella \& Natta (2005), a completely vertical rim will produce no excess flux if faceon, but they argue that the dependence of the grain sublimation temperature on gas density will result in a curved rim, which will produce excess flux even if face-on. In the regions probed by the millimeter observations, which are far from the dust sublimation radius, an inner rim which is vertically extended will have a higher temperature and will therefore produce additional flux (see Calvet et al. 2002). Here, we empirically add excess flux in a thin ring at the inner rim. The ratio of the excess flux to the flux from a standard flared disk at that radius is controlled by a free parameter in our model and we assume a $10 \%$ width of the rim. The range of this model parameter is from 1 (no excess) to 5 and is meant to incorporate emission from both the optically thick and thin portions of the rim.

\subsection{Optically Thin Material and Scattering}

The measured accretion rate (Muzerolle et al. 2000; Eisner et al. 2010) implies that no matter what the structure of the 
Table 3

Model Parameters

\begin{tabular}{|c|c|c|c|c|}
\hline Parameter & $\begin{array}{c}\text { Example Model: } \\
\text { VLA+SED Fit }\end{array}$ & $\begin{array}{l}\text { Example Model: } \\
\text { VLTI+SED Fit }\end{array}$ & $\begin{array}{c}\text { Example Model: } \\
\text { All Data Fit }\end{array}$ & $\begin{array}{l}\text { Ranges for } \\
\text { All Data Fit }\end{array}$ \\
\hline \multicolumn{5}{|l|}{ Optically thin emission: } \\
\hline Inner radius (AU) & 0.02 & 0.02 & 0.02 & Not constrained \\
\hline$\Sigma_{0}\left(\mathrm{~g} \mathrm{~cm}^{-} 2\right)$ & $1 \times 10^{-8}$ & $1 \times 10^{-8}$ & $1 \times 10^{-8}$ & $<1 \times 10^{-8}$ \\
\hline$\beta$ & 1 & 1 & 1 & Not constrained \\
\hline$C_{\text {scat }}$ & $1 \times 10^{-4}$ & $1 \times 10^{-4}$ & $1 \times 10^{-4}$ & $8 \times 10^{-5}-1.1 \times 10^{-4}$ \\
\hline \multicolumn{5}{|c|}{ Inner optically thick disk: } \\
\hline Inner radius (AU) & $\cdots$ & 0.3 & 0.51 & $0.42-0.52$ \\
\hline Radial extent (AU) & $\cdots$ & 70 & 0.13 & $<0.13$ \\
\hline$\Sigma_{0}\left(\mathrm{~g} \mathrm{~cm}^{-2}\right)$ & $\cdots$ & 90 & 3 & $>0.1$ \\
\hline$\beta$ & $\cdots$ & 0.6 & -0.03 & $-0.15-0.1$ \\
\hline$f_{\text {rim }}$ & $\cdots$ & 5 & 1.25 & $1-1.5$ \\
\hline \multicolumn{5}{|c|}{ Outer optically thick disk: } \\
\hline Inner radius (AU) & 2.9 & $\cdots$ & 4.5 & $3.8-4.5$ \\
\hline Radial extent (AU) & 70 & $\cdots$ & 70 & $70-75$ \\
\hline$\Sigma_{0}\left(\mathrm{~g} \mathrm{~cm}^{-2}\right)$ & 140 & $\cdots$ & 180 & $110-180$ \\
\hline$\beta$ & 1.0 & $\cdots$ & 0.95 & $0.95-1.15$ \\
\hline$f_{\text {rim }}$ & 3.3 & $\cdots$ & 1.75 & $1-1.75$ \\
\hline$\chi_{v}^{2}$ & 60 & 120 & 14 & \\
\hline
\end{tabular}

optically thick disk is, there is some flow of material onto the central star. The near-IR interferometry data are the best tracer of that emission in the data considered here. However, as discussed in Section 2.3, the near-IR interferometry data also suggest that a scattering component is present as well. For the inner disk, we use a model with an optically thin component and include a contribution from scattering from the entire disk. The parameters for the optically thin emission are the inner edge of the optically thin dust (constrained to be larger than the dust sublimation radius), the surface density (required to be optically thin), and the dust opacity frequency exponent. The optically thin dust is assumed to extend to the inner edge of the optically thick dust, but this is not well constrained by the data. The mass opacity law for the optically thin emission is taken to be $\kappa_{\lambda}=\kappa_{0}\left(\frac{\lambda}{\lambda_{0}}\right)^{-\beta}$.

Weinberger et al. (2002) measured scattering in the outer disk of TW Hya (20-200 AU) and found scattered light contributions of $2.4 \%$ and $2.1 \%$ at 1.1 and $1.6 \mu \mathrm{m}$ over these radii. For our model, we extrapolate using the Weinberger et al. (2002) values as they observed closest to the central star, but observations by Trilling et al. (2001) found a similar level for the scattered flux level at these wavelengths. Weinberger et al. (2002) showed that the scattered light surface brightness profile was flat within $40 \mathrm{AU}$ and scales as $r^{-2.6}$ for radii greater than $40 \mathrm{AU}$. This radial profile is intermediate between those predicted for a geometrically flat disk $\left(r^{-3}\right)$ and a flared disk $\left(r^{-2}\right.$; Whitney \& Hartmann 1992).

For the scattering component in our model, we adopt the radial profile measured by Weinberger et al. (2002) and assume it also applies at radii less than $20 \mathrm{AU}$. We assume that the scattering flux goes as $\lambda^{-1}$ for wavelengths longer than the $H$ band. This relation is close to the scattered flux wavelength dependence in the near-IR found by Whitney et al. (1997) in modeling Taurus sources. Scattered light observations over longer wavelengths are necessary to constrain this dependence. As the scattered flux is smaller than the uncertainties in the disk/stellar flux ratio at these wavelengths, this assumption only impacts the parameters of the optically thin portion of the disk. Extrapolating to the $K$ band using this relation gives a total scattered flux of $1.5 \% \pm$ $0.15 \%$ outside of $20 \mathrm{AU}$. The scattered flux as a function of radius is then defined as

$$
\begin{gathered}
F_{\text {scat }}(r)=F_{*} C_{\text {scat }} 2 \pi r d r ; \text { for } r<40 \mathrm{AU} \\
F_{\text {scat }}(r)=F_{*} C_{\text {scat }} 2 \pi r^{-1.6} d r ; \text { for } r>40 \mathrm{AU},
\end{gathered}
$$

where $F_{*}$ is the stellar flux at the $K$ band $(0.74 \mathrm{Jy})$ and $C_{\text {scat }}$ is the scaling factor included as a model parameter. Scattering is included only at radii where disk material is present. Due to the $r^{-2.6}$ scaling, very little scattered light would arise from beyond our outer disk cutoff.

In addition to the KI and CHARA interferometry data, we use the extrapolated $K$-band total scattering outside of $20 \mathrm{AU}$ as an additional constraint. In the fits to the $2 \mu \mathrm{m}$ interferometry and the scattered flux data, the inner radius and the mass opacity law are not constrained. We set the inner radius at the dust sublimation radius, as the observed accretion suggests at least some disk material is present this close to the star. Although there may be near-IR emission from within this radius, we do not have sufficient spatial resolution to resolve this. The exponent for the opacity law is not constrained by these data and a value of $\beta=1$ is used, which is the value for the grain size and composition used by CG97 models. The constraints for the surface density and scattering coefficient are given in the last column of Table 3. The parameters for the optically thin disk and scattering are held constant while fitting for the outer disk parameters as the near-IR interferometry data do not constrain the parameters of the outer disk, other than excluding the inner edge of the optically thick disk from values near the dust sublimation radius. As can be seen in Figures 4-6, even with this scattering component, the model still somewhat overestimates the visibility on the shortest baseline and so our assumption that the scattering surface brightness is flat within 40 AU may underestimate the scattering within the CHARA FOV (32 AU radius). However, data at additional wavelengths and spatial scales are necessary to test our assumption of a flat surface brightness profile due to scattering within 20 AU.

\subsection{Single Flared Disk}

As demonstrated by Ratzka et al. (2007) and Hughes et al. (2007), a flared disk plus optically thin inner emission can be 

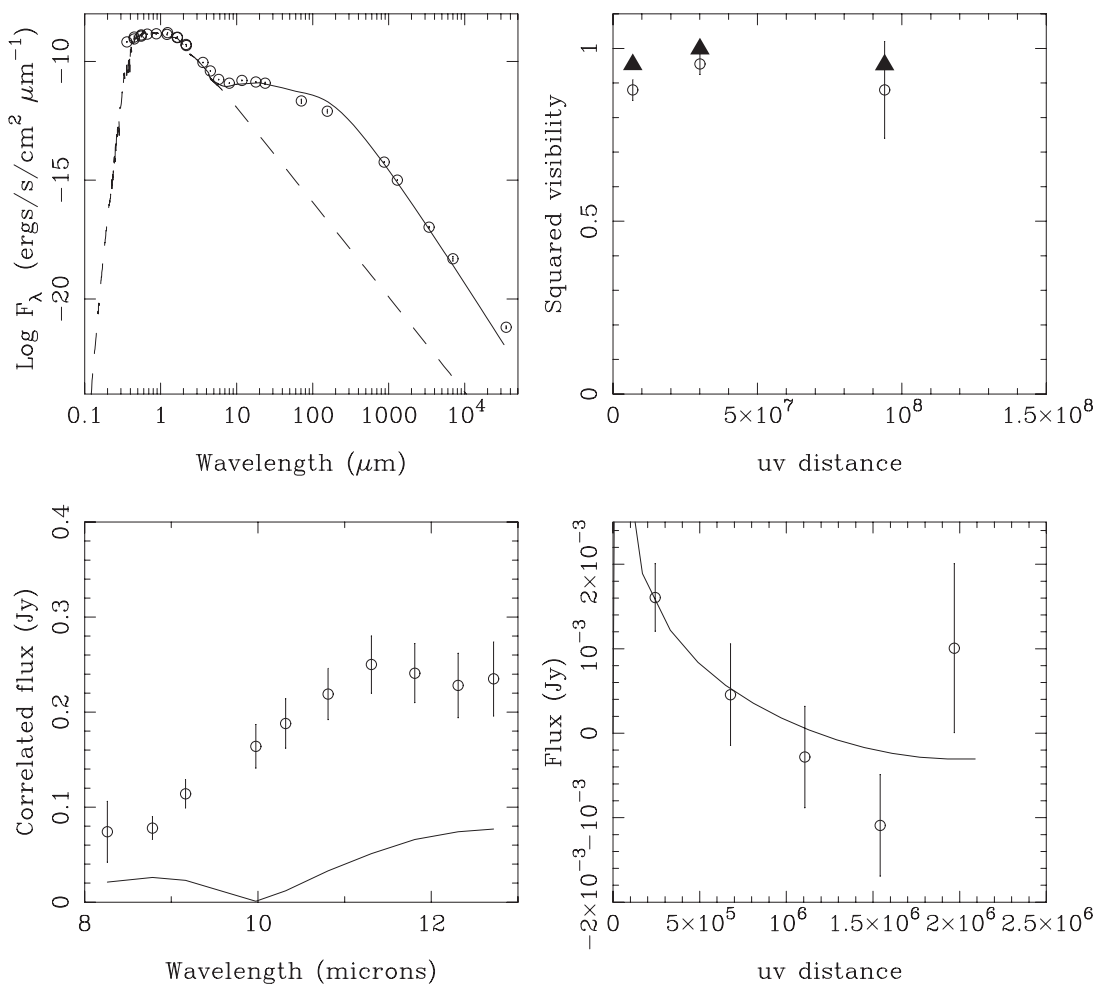

Figure 4. Example model with a single flared disk and optically thin emission where the parameters have been optimized to fit the VLA and SED data. The top left panel shows the SED data, stellar model (dashed line), and disk model (solid line). The bottom left panel shows the correlated flux from VLTI at $10 \mu \mathrm{m}$ from Ratzka et al. (2007) and the model (solid line). The top right panel shows the KI and CHARA squared visibilities (circles) and the model visibilities (filled triangles) which have been filtered by the appropriate field of view for the given telescope. The bottom right panel shows the VLA visibility amplitude at $7 \mathrm{~mm}$ from Hughes et al. (2007) and the model (solid line). See Table 3 for the model parameter values.

used to fit either the $7 \mathrm{~mm}$ VLA and SED data or the VLTI and SED data. In addition, Ratzka et al. (2007) also fit the Eisner et al. (2006) KI data point. However, using either of these models to reproduce the remaining spatially resolved data does not produce a good fit.

For example, Figure 4 shows a model which is a very good fit to the $7 \mathrm{~mm}$ and SED data with an inner radius of $2.9 \mathrm{AU}$, a flux enhancement of 3.3 at the inner rim, and optically thin emission from the inner rim inward to $0.02 \mathrm{AU}$. However, this model completely fails to reproduce the VLTI data (lower left panel) as the radial profile is dominated by emission on much larger scales (a few AU) than the 0.4-0.7 AU scales suggested by the geometric fits (Section 2.3). The model parameters used for Figure 4 are given in Table 3 along with $\chi_{v}^{2}$ for the complete data set.

A similar fit was also tried for the combination of the VLTI and SED data. Although a single flared disk with extended inner rim can roughly match both the VLTI and SED data, the fit to either component is not as good as the fit to both components for the VLA/SED fit. An example model is shown in Figure 5. The inner radius of the optically thick disk in this model (0.3 AU) is smaller than the radius found by Ratzka et al. (2007) as the flux from larger scales in the flared disk must be balanced by emission from small radii to produce the partially resolved visibilities. We note that the Ratzka et al. (2007) model does not have a vertically extended inner rim, but instead has an radially extended transition zone from the optically thin inner emission to the inner edge of an optically thick flared disk, which produces emission from a wider range of radii than our model which assumes a rim width of $10 \%$. The Ratzka et al. (2007) model produces a better fit to the VLTI data than our single disk model.
In the single disk model optimized for the VLTI data, the predicted $7 \mathrm{~mm}$ visibilities are too high compared to the VLA data. As there are no features on the spatial scales predicted by the $7 \mathrm{~mm}$ interferometry data ( $\sim 4 \mathrm{AU})$, the model visibility curve can only be changed by increasing or decreasing the total flux which is set by the surface density and emissivity. However, decreasing the total $7 \mathrm{~mm}$ flux also decreases the total flux at all millimeter wavelengths which does not match the long wavelength data in the SED. This can be seen by comparing the model of Hughes et al. (2007), which has $1.7 \mathrm{mJy}$ of flux in the resolved observations, to the total $7 \mathrm{~mm}$ flux of $8 \pm 1 \mathrm{mJy}$ measured by Wilner et al. (2000).

\subsection{Flared Disk with Gap}

The next addition to the model was to break the optically thick disk into two components. Each component has a separate set of parameters (inner radius, radial extent, surface density, and opacity wavelength dependence). The optically thin component remains tied to the inner radius of the inner optically thick disk. Figure 6 shows an example model which reproduces all four data sets and Table 3 gives the values for each parameter which correspond to a $67 \%$ probability range. As seen by the values for $\chi_{v}^{2}$, although this model does not exactly reproduce the data within the uncertainties, the fit is substantially improved over the single disk models.

The spatially distinct features (two inner edges) of this model allow the $10 \mu \mathrm{m}$ and $7 \mathrm{~mm}$ interferometry data, which probe flux as a function of spatial frequency, to be fit much more precisely than a single disk. The cumulative radial flux profile at $10 \mu \mathrm{m}$ and $7 \mathrm{~mm}$ is shown in Figure 7, showing the contribution at each wavelength. The inner ring dominates at $10 \mu \mathrm{m}$, while 

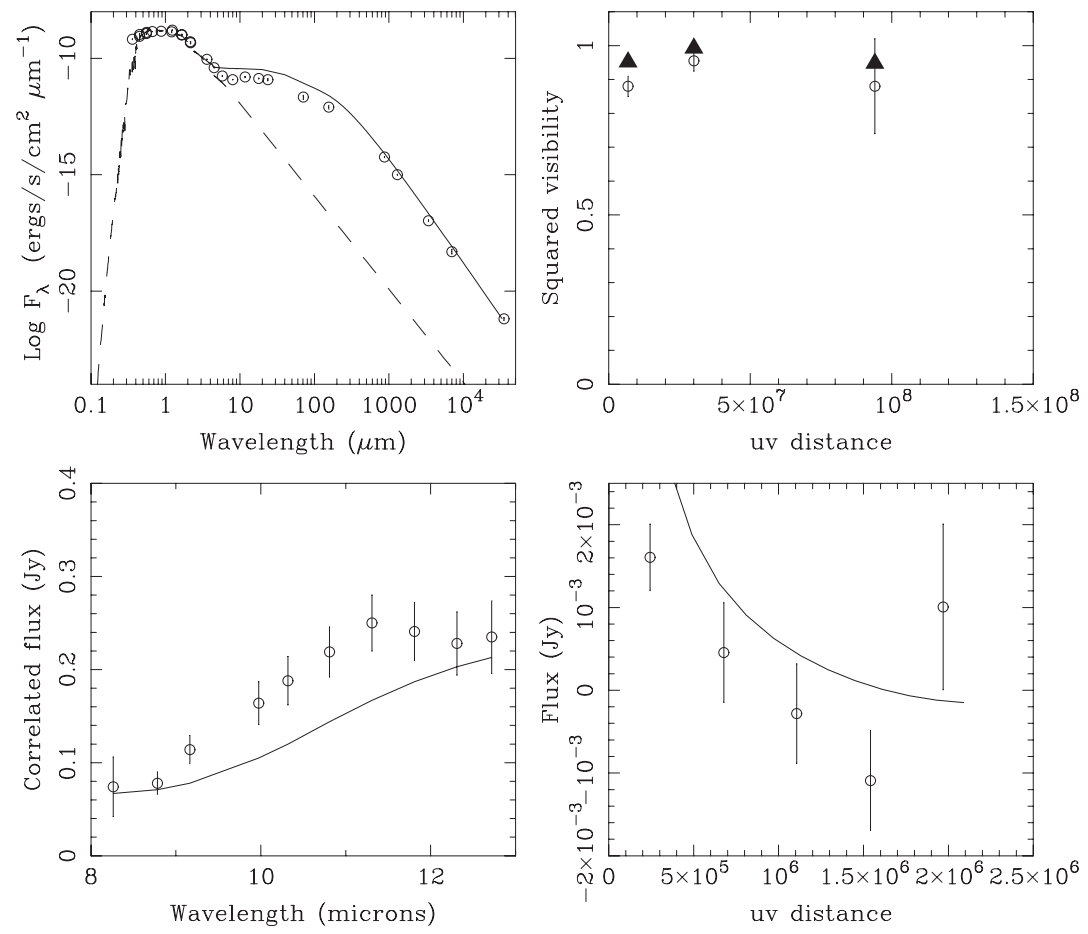

Figure 5. As for Figure 4, but for a single disk model where the parameters have been optimized to fit the VLTI and SED data. See Table 3 for the model parameter values.
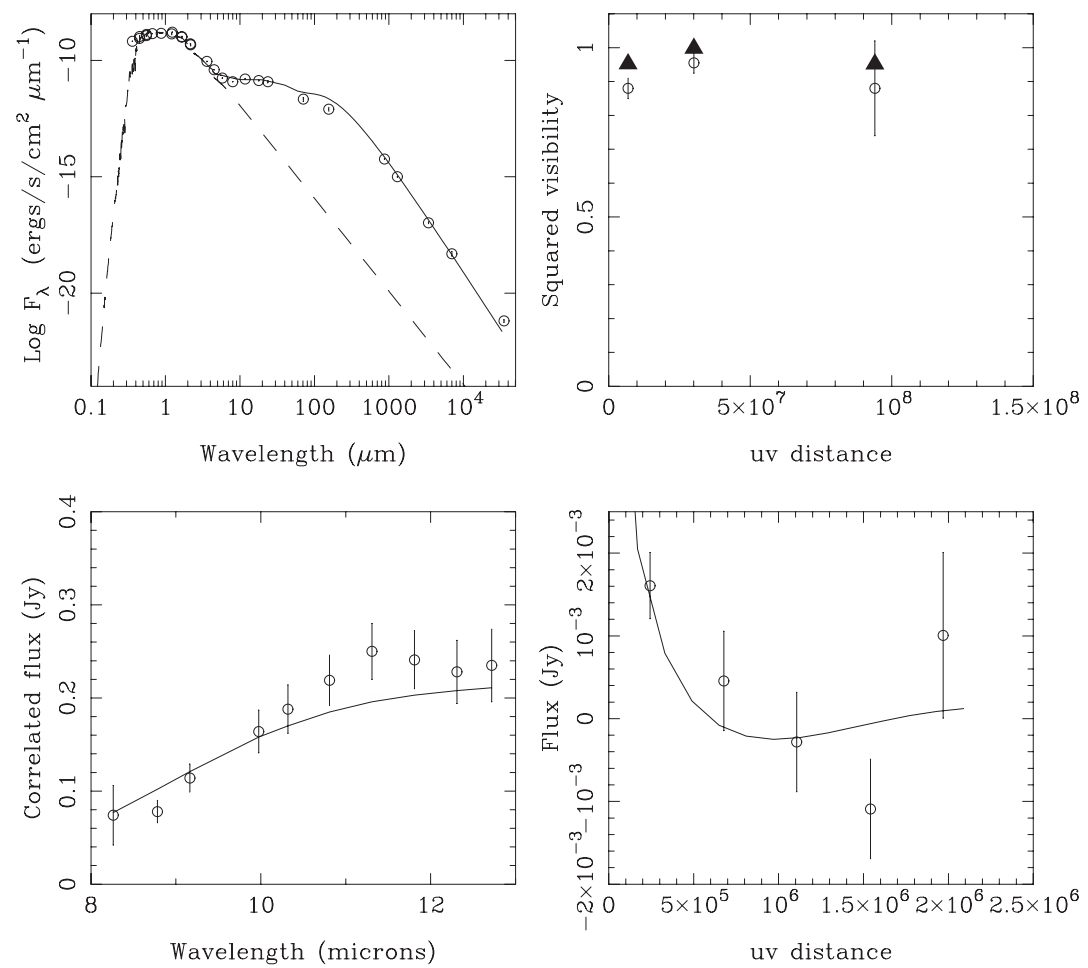

Figure 6. As for Figure 4, but for a model with an inner and outer optically thick disk. See Table 3 for the model parameter values.

the outer ring dominates at $7 \mathrm{~mm}$. The inflection in the outer ring at $35 \mathrm{AU}$ is due to the change in the temperature profile (Equation (3)).

The inner optically thick disk has a radial extent less than $1 \mathrm{AU}$ and thus is probably better described as a ring. With only a single baseline of mid-IR data, it is not possible to constrain if the material is asymmetric or clumpy. The optical depth of the inner ring is not well constrained. It must have $\Sigma_{0} \gtrsim 0.1 \mathrm{~g} \mathrm{~cm}^{-2}$ at $1 \mathrm{AU}$, such that the mid-IR emission is optically thick, but the surface area is so small compared with the outer disk that it has a negligible effect on the long-wavelength flux. In particular, the inner disk may have a surface density as high as that of the outer disk $\left(\sim 100 \mathrm{~g} \mathrm{~cm}^{-2}\right.$ at $\left.1 \mathrm{AU}\right)$. The inner radius of the inner ring is degenerate with the value of $\beta$ for our model, with smaller values of $r_{\text {in }}$ corresponding to higher values of $\beta$. The flux excess at the inner rim is low (1-1.5), making the inner ring more like the gradual transition to optically thick material as proposed by Ratzka et al. (2007) than a standard vertically extended inner 

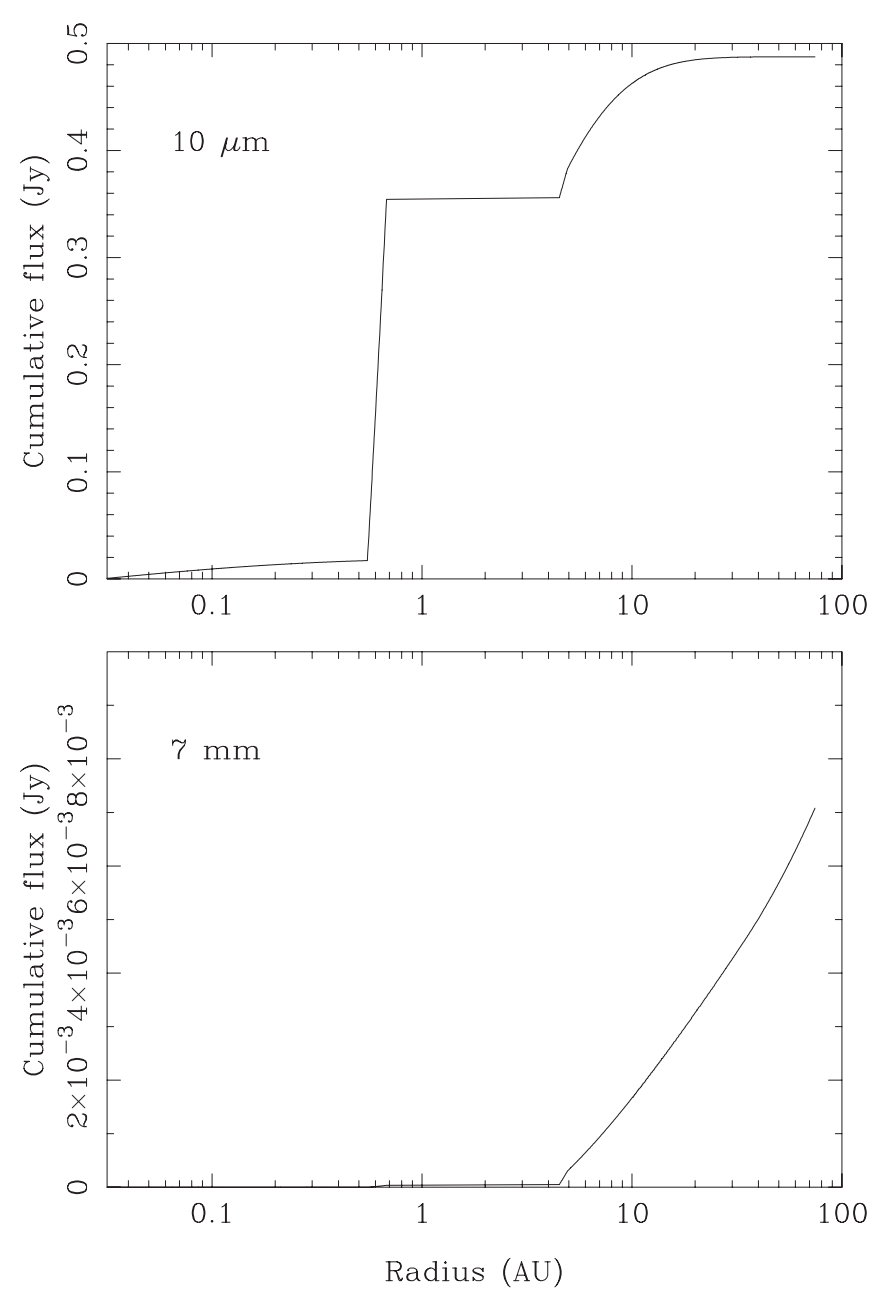

Figure 7. Cumulative flux as a function of radius at $10 \mu \mathrm{m}$ (top) and $7 \mathrm{~mm}$ (bottom) for the disk model shown in Figure 6.

rim. The radial extent of the inner ring is strongly constrained to be less than $0.2 \mathrm{AU}$, but we did not explore values lower than $0.1 \mathrm{AU}$ as our formulation for the inner rim flux enhancement then becomes degenerate with the surface density.

The outer ring is very similar to the disk fit to TW Hya by Calvet et al. (2002) and Hughes et al. (2007). The range of inner rim radii of our model (3.8-4.5 AU) includes their derived values and the value of $\beta$ found for the outer disk $(0.95-1.15)$ is similar to the value of 0.8 used by Hughes et al. (2007).

\section{DISCUSSION}

The model presented here is not a unique solution to fitting these data, especially as some parameters were set with prior information or held fixed, but the failure of a continuous, flared disk to reproduce these data sets is clear. The main result of this work is that it is possible to find a disk morphology that reproduces the observed spatially resolved data and the SED. This model combines elements of previous work, particularly the optically thick disks at $\sim 0.5$ and 4 AU. Unlike previous models, there is a substantial decrease in the surface density after the $0.5 \mathrm{AU}$ disk. As the surface density of the inner disk is not well constrained, this structure could either be considered as a gap within the optically thick disk or if the inner $0.5 \mathrm{AU}$ disk is at a much lower surface density than the outer disk, the 0.5 AU material may represent an optically thick ring within the inner gap of the $4 \mathrm{AU}$ disk. In either case, some process is necessary to produce the opacity decrease between the inner and outer optically thick disks.

Some transition disk objects have been revealed to be close binaries where the inner disk gap is due to clearing by a stellar companion rather than disk evolution (e.g., CoKu Tau 4; Ireland \& Kraus 2008). In TW Hya, a stellar companion at a few AU ( 40 mas) would clear the circumbinary disk to $\sim 4$ AU (Artymowicz \& Lubow 1994) and the mid-IR material could reside in a circumstellar disk. However, there is no evidence for stellar multiplicity in this system. A radial velocity survey of the TW Hya association spanning 18 years (Torres et al. 2003) detected no stellar companions for TW Hya and the $\sim 200 \mathrm{~m} \mathrm{~s}^{-1}, 3$ day periodicity in optical radial velocity detected by Setiawan et al. (2008) was shown by Huélamo et al. (2008) to be more likely due to a stellar spot and even if due to a planet, the orbital radius of $0.04 \mathrm{AU}$ would not affect the disk structure at 4 AU. TW Hya was included in an adaptive optics imaging survey by Brandeker et al. (2003) with no detections from 0.042 to $1^{\prime \prime} 6$ (2.2-86 AU). Thus, it is unlikely that the proposed gap in the optically thick disk is due to a stellar companion.

Of the mechanisms proposed for disk clearing in single stars (planet formation, grain growth, photoevaporation), the presence or formation of a planet is the strongest candidate for gap formation in an optically thick gas and dust disk. Grain growth has been shown to be capable of producing rings in optically thin debris disks (Takeuchi \& Artymowicz 2001; Kenyon \& Bromley 2004), but these disks have very different dynamics than an optically thick gas and dust disk like our TW Hya model. Gap clearing in disks was first explored by Lin \& Papaloizou (1979) and Artymowicz \& Lubow (1994) and the mass ratio of the two bodies is a crucial parameter in determining the size and effectiveness of the clearing. A larger body will produce a larger and more cleared gap, and thus characterizing the properties of the gap helps to constrain the possible formation mechanisms.

One aspect of our model is that the outer disk has enhanced emission at the inner edge. Disk models with vertically extended rims at the dust sublimation radius are now widely accepted to fit near-IR interferometry measurements and the so-called Herbig SED bump (see review by Dullemond \& Monnier 2010, and references therein). The enhanced emission at $\sim 4 \mathrm{AU}$ in our model could arise either from a vertically extended structure or from an increase in the surface density at this radius. To directly measure the structure of the inner rims at 0.5 and $4 \mathrm{AU}$, resolved observations on longer baselines are needed in the mid-IR and millimeter. Espaillat et al. (2010) have calculated that stellar flux can illuminate the outer disk rim in pre-transitional disks (see Section 4.1) due to the finite size of the star itself. The shadow cast by the star depends on the height of the inner disk rim. We note that the flux increase at the rim of the inner optically thick disk in our model is low (1-1.5) and thus the vertical extension could be small enough such that a substantial fraction of the outer disk rim is directly illuminated resulting in higher temperatures and increased flux.

As the main observational constraint for increased emission at the inner rim of the outer disk comes from the $7 \mathrm{~mm}$ data, where the dust emission is optically thin, the second option for producing extra emission at the inner rim would be to increase the surface density. Evidence for increased surface density past a planet-formed gap is seen in the models of Bryden et al. (1999). In their models, a pile-up of material beyond the planet results in a thin ring on the outer edge of the gap where the surface 
density is roughly two times higher. This factor is consistent with the range of flux ratios needed in our model (1-1.75) to fit the $7 \mathrm{~mm}$ data.

If a planet is producing a gap between the inner and outer disk, the dust properties in the inner and outer disks are likely to be different. Rice et al. (2006) found that the planet produces a pressure filter in which larger particles are kept outside of the planet's orbital radius. The values of $\beta$ for the inner and outer optically thick disks in our model are actually opposite this prediction. However, given that the inner disk model parameters are dominated by fitting data from 8 to $13 \mu \mathrm{m}$ and the value of $\beta$ in the inner disk is degenerate with other disk parameters such as the width of the ring, we do not consider the difference in $\beta$ to be well constrained.

Another observational test of which physical mechanism could produce a gap is to look at gas in the disk. While the observations modeled here probe only the dust mass, many observations exist which probe the gas. Pontoppidan et al. (2008) used the $4.7 \mu \mathrm{m}$ rovibrational lines of $\mathrm{CO}$ to probe the gas in the inner disk region. They found the gas extended from $0.11 \pm 0.07 \mathrm{AU}$ to $>1.5 \mathrm{AU}$ by fitting Keplerian rotation to the measured gas velocity profiles. The outer cutoff is not well determined by their data as the emission may decrease due to cooler temperatures rather than lack of gas. Najita et al. (2010) used Spitzer spectroscopy at mid-IR wavelengths to probe the gas disk. Unlike other T Tauri disks, the TW Hya disk did not have strong features from $\mathrm{H}_{2} \mathrm{O}, \mathrm{C}_{2} \mathrm{H}_{2}$, or $\mathrm{HCN}$, but did show atomic emission such as $\mathrm{H}$ I and $\mathrm{Ne}$ and molecular emission from $\mathrm{H}_{2}, \mathrm{OH}$, and $\mathrm{CO}_{2}$. Given the excitation temperatures of these transitions, they infer that the inner disk $(<5 \mathrm{AU})$ has undergone significant chemical evolution, assuming TW Hya once looked like a typical T Tauri disk. As discussed by Najita et al. (2010), this reduced emission is consistent with clearing by an orbiting giant planet or may be due to chemical evolution or an excitation effect. Simulations by Paardekooper \& Mellema (2004) found that a $1 M_{\text {Jupiter }}$ planet will clear a gap in both the gas and dust disk while a $0.1 M_{\text {Jupiter }}$ planet will only clear the dust. If the gap between the inner and outer disk is due to clearing by a planet, the detection of gas within the $4 \mathrm{AU}$ outer disk may constrain the mass of that planet.

If the gap is produced by a planet, the radial extent of the optically thick dust brackets its likely location. Given this, we can calculate the corresponding range of periods and radial velocity signatures. For simplicity in our circumstellar disk modeling, we assumed an inclination of $0^{\circ}$, which would produce no radial velocity signature if the planet orbital plane and disk were co-aligned. To calculate potential planetary signatures, we used the inclination of $7^{\circ}$ assumed by Huélamo et al. (2008). Table 4 gives the period, radial velocity, and astrometric signature for a range of planet masses at radii close to the inner ring, the inner rim of the outer disk, and an intermediate value. $\mathrm{T}$ Tauri stars have significant stellar activity making radial velocities measurements inherently more noisy than observations of main-sequence stars. In the observations of Setiawan et al. (2008), the uncertainty on the TW Hya data was $40 \mathrm{~m} \mathrm{~s}^{-1}$, while a quiet star observed with the same instrument setup had an uncertainty of $5 \mathrm{~m} \mathrm{~s}^{-1}$. Unless the stellar activity signatures in the radial velocities can be removed, the radial velocity signatures predicted in Table 4 would not be detectable toward TW Hya. A potentially better detection method for planets at these radii around young stars is astrometry. Currently both KI and the VLTI are developing astrometric capability with predicted performance levels of 25-100 $\mu$ as which will
Table 4

Predicted Planet Signatures

\begin{tabular}{lccccccc}
\hline \hline $\begin{array}{c}\text { Orbital Radius } \\
(\mathrm{AU})\end{array}$ & $\begin{array}{c}\text { Period } \\
(\mathrm{yr})\end{array}$ & \multicolumn{3}{c}{$\begin{array}{c}\text { Radial Velocity } \\
\left(\mathrm{m} \mathrm{s}^{-1}\right)\end{array}$} & \multicolumn{3}{c}{$\begin{array}{c}\text { Astrometric Signature } \\
(\mu \text { as })\end{array}$} \\
\hline 0.5 & & $0.1 M_{J}$ & $1 M_{J}$ & $5 M_{J}$ & $0.1 M_{J}$ & $1 M_{J}$ & $5 M_{J}$ \\
2.75 & 0.5 & 0.7 & 6.7 & 33 & 25 & 250 & 1300 \\
5 & 6 & 0.3 & 3.0 & 15 & 140 & 1400 & 7000 \\
& 14 & 0.2 & 2.4 & 12 & 250 & 2500 & 13000 \\
\hline
\end{tabular}

be available in the next few years (Delplancke 2008; Woillez et al. 2010). A planet is detectable at these levels for all but the smallest orbits or masses and a potential reference star is nearby, making TW Hya a strong candidate for astrometric planet searches.

\subsection{Implications of a Gap for the Evolutionary State of TW Hya}

Espaillat et al. (2007) proposed a new class of transition disks, the "pre-transitional disks" which have evidence for optically thick emission close to the star, a region of lower dust opacity and an outer, optically thick disk. In a study of three such objects (LkCa 15, UX Tau A, and ROX 44), Espaillat et al. (2010) found the optically thick inner portion began at a few tenths of AU and extended up to 4 AU. Like these "pre-transitional" disks, our disk model for TW Hya contains a gap in the optically thick portion of the disk. However, in these sources, the inner disk starts at the dust sublimation radius, unlike our model for TW Hya, and the disk gaps, which ranged from 20 to $70 \mathrm{AU}$, are much larger than the gap of 3-4 AU in our model for TW Hya.

In the disk clearing models of Alexander \& Armitage (2007) after a gap has formed due a planet, there is a stage where the inner disk still exists, but has a surface density orders of magnitude less than the outer disk. However, this stage is relatively short-lived in their models $\left(<10^{5} \mathrm{yr}\right)$ and given the age of TW Hya, it is unlikely that planet formation has taken place this recently. Radial gaps within disk are also found in much older debris disks, including eps Eri (Backman et al. 2009) and beta Leo (Akeson et al. 2009).

An additional aspect of our disk model which may have bearing on the evolutionary state is that the innermost optically thick region does not start at the dust sublimation radius. For a dust sublimation temperature of $1500 \mathrm{~K}$, and assuming optically thin dust, the sublimation radius for $1 \mu \mathrm{m}$ sized grains is $0.02 \mathrm{AU}$. This is considerably closer to the star than the 0.42-0.52 AU for the inner radius of the inner optically thick portion. In considering the mass and accretion rate of the entire disk, Alexander \& Armitage (2007) found that inner hole in the TW Hya disk was unlikely to be due to photoevaporation. However, if the gap between the inner and outer disk is formed by a planet, the flow of material to the inner disk will be much lower, although probably not zero, as streams of material may cross this gap (Artymowicz \& Lubow 1994). Thus, it may be possible that the inner disk is sufficiently influenced by photoevaporation to affect the inner dust radius. However, this is again a short-lived phase in the evolution of a disk (see, e.g., Alexander et al. 2006).

\section{CONCLUSIONS}

We have presented new near-IR interferometry observations of the TW Hya disk that show that the near-IR flux within $30 \mathrm{AU}$ of the star contains a substantial component that is overresolved 
on a $15 \mathrm{~m}$ baseline. This is most likely an extension of the scattered flux observed on larger spatial scales. We combined these data with previously published spatially resolved data and found a physically plausible disk model for TW Hya which reproduces spatially resolved data at $2 \mu \mathrm{m}, 10 \mu \mathrm{m}$, and $7 \mathrm{~mm}$ and the SED. Our model follows previous work in using a disk structure with optically thin emission interior to an optically thick disk and includes emission at spatial scales previously proposed (0.5 AU and 4 AU; Calvet et al. 2002; Ratzka et al. 2007; Hughes et al. 2007), but unlike the previous models, we find an opacity gap between the inner and outer optically thick disks. Our model is not a unique fit to the data; in particular, we did not investigate whether substantial radial changes in density and dust opacity could also reproduce the data sets considered here; e.g., a region of low emissivity instead of a gap, followed by a large increase in the surface density or dust opacity at $4 \mathrm{AU}$. However, any model with flux at 0.5 and 4.0 AU to match resolved observations at $10 \mu \mathrm{m}$ and $7 \mathrm{~mm}$ needs substantial radial structure or discontinuities and therefore would require a driving mechanism for that radial structure. The model described here is consistent with the formation of a planet within the disk as first proposed by Calvet et al. (2002). Although we cannot prove the existence of such a planet, a gap within an optically thick disk can be produced by planet formation but not by photoevaporation, which can only form inner holes. Further observations are needed to resolve the radial structure of the inner disk. Near-IR interferometric imaging would constrain the distribution of the optically thin material while mid-IR interferometric imaging over a range of baselines would constrain the inner disk and possibly the existence of a gap. In any case, the TW Hya disk contains more structure in the central region than previously suggested and, due to its proximity to Earth and its age, will continue to serve as one of the best-studied examples of disk structure and evolution.

We thank the CHARA staff, particularly C. Farrington, for their excellent help in obtaining the data. We thank Meredith Hughes for providing data and Geoff Bryden for helpful discussions and the anonymous referee for suggestions which improved the paper. The CHARA Array is funded by the National Science Foundation through NSF grant AST-0908253, by Georgia State University through the College of Arts and Sciences, and by the W. M. Keck Foundation. The Keck Interferometer is funded by the National Aeronautics and Space Administration as part of its Exoplanet Exploration program. This work was performed at the NASA Exoplanet Science Institute (NExScI), Caltech and made use of the SIMBAD database, operated at CDS, Strasbourg, France and the NASA Star and Exoplanet Database (NStED) at the Infrared Processing and Analysis Center and services at NExScI. NStED is jointly funded by the National Aeronautics and Space Administration (NASA) via Research Opportunities in Space Sciences grant 2003 TPF-FS, and by NExScI.

Facilities: CHARA, Keck: Interferometer

\section{REFERENCES}

Akeson, R. L., et al. 2009, ApJ, 691, 1896

Alexander, R. D., \& Armitage, P. J. 2007, MNRAS, 375, 500

Alexander, R. D., Clarke, C. J., \& Pringle, J. E. 2006, MNRAS, 369, 229

Artymowicz, P., \& Lubow, S. H. 1994, ApJ, 421, 651

Backman, D., et al. 2009, ApJ, 690, 1522

Brandeker, A., Jayawardhana, R., \& Najita, J. 2003, AJ, 126, 2009
Bryden, G., Chen, X., Lin, D. N. C., Nelson, R. P., \& Papaloizou, J. C. B. 1999, ApJ, 514, 344

Calvet, N., D’Alessio, P., Hartmann, L., Wilner, D., Walsh, A., \& Sitko, M. 2002, ApJ, 568, 1008

Chiang, E. I., \& Goldreich, P. 1997, ApJ, 490, 368

Colavita, M., et al. 2003, ApJ, 592, L83

D’Alessio, P., Calvet, N., \& Hartmann, L. 2001, ApJ, 553, 321

Delplancke, F. 2008, New Astron. Rev., 52, 199

Dullemond, C. P., Dominik, C., \& Natta, A. 2001, ApJ, 560, 957

Dullemond, C. P., \& Monnier, J. D. 2010, ARA\&A, 48, 205

Eisner, J. A., Chiang, E. I., \& Hillenbrand, L. A. 2006, ApJ, 637, L133

Eisner, J. A., Doppmann, G. W., Najita, J. R., McCarthy, D., Kulesa, C., Swift, B. J., \& Teske, J. 2010, ApJ, 722, 28

Espaillat, C., Calvet, N., D’Alessio, P., Hernández, J., Qi, C., Hartmann, L., Furlan, E., \& Watson, D. M. 2007, ApJ, 670, L135

Espaillat, C., et al. 2010, ApJ, 717, 441

Figueira, P., et al. 2010, A\&A, 511, A55

Furlan, E., et al. 2009, ApJ, 703, 1964

Hartmann, L., Megeath, S. T., Allen, L., Luhman, K., Calvet, N., D’Alessio, P., Franco-Hernandez, R., \& Fazio, G. 2005, ApJ, 629, 881

Herczeg, G. J., Wood, B. E., Linsky, J. L., Valenti, J. A., \& Johns-Krull, C. M. 2004, ApJ, 607, 369

Hoff, W., Henning, T., \& Pfau, W. 1998, A\&A, 336, 242

Huélamo, N., et al. 2008, A\&A, 489, L9

Hughes, A. M., Wilner, D. J., Calvet, N., D’Alessio, P., Claussen, M. J., \& Hogerheijde, M. R. 2007, ApJ, 664, 536

Ireland, M. J., \& Kraus, A. L. 2008, ApJ, 678, L59

Isella, A., Carpenter, J. M., \& Sargent, A. I. 2009, ApJ, 701, 260

Isella, A., \& Natta, A. 2005, A\&A, 438, 899

Johns-Krull, C. M., \& Valenti, J. A. 2001, ApJ, 561, 1060

Kenyon, S. J., \& Bromley, B. C. 2004, ApJ, 602, L133

Lin, D. N. C., \& Papaloizou, J. 1979, MNRAS, 186, 799

Low, F. J., Smith, P. S., Werner, M., Chen, C., Krause, V., Jura, M., \& Hines, D. C. $2005, \mathrm{ApJ}, 631,1170$

Merín, B., et al. 2010, ApJ, 718, 1200

Muzerolle, J., Allen, L. E., Megeath, S. T., Hernández, J., \& Gutermuth, R. A. 2010, ApJ, 708, 1107

Muzerolle, J., Calvet, N., Briceño, C., Hartmann, L., \& Hillenbrand, L. 2000, ApJ, 535, L47

Najita, J. R., Carr, J. S., Strom, S. E., Watson, D. M., Pascucci, I., Hollenbach, D., Gorti, U., \& Keller, L. 2010, ApJ, 712, 274

Najita, J. R., Strom, S. E., \& Muzerolle, J. 2007, MNRAS, 378, 369

Natta, A., Prusti, T., Neri, R., Wooden, D., Grinin, V. P., \& Mannings, V. 2001, A\&A, 371, 186

Paardekooper, S.-J., \& Mellema, G. 2004, A\&A, 425, L9

Pontoppidan, K. M., Blake, G. A., van Dishoeck, E. F., Smette, A., Ireland, M. J., \& Brown, J. 2008, ApJ, 684, 1323

Qi, C., et al. 2004, ApJ, 616, L11

Ratzka, T., Leinert, C., Henning, T., Bouwman, J., Dullemond, C. P., \& Jaffe, W. 2007, A\&A, 471, 173

Rice, W. K. M., Armitage, P. J., Wood, K., \& Lodato, G. 2006, MNRAS, 373, 1619

Rucinski, S. M., \& Krautter, J. 1983, A\&A, 121, 217

Rucinski, S. M., et al. 2008, MNRAS, 391, 1913

Setiawan, J., Henning, T., Launhardt, R., Müller, A., Weise, P., \& Kürster, M. 2008, Nature, 451, 38

Strom, K. M., Strom, S. E., Edwards, S., Cabrit, S., \& Skrutskie, M. F. 1989, AJ, 97, 1451

Takeuchi, T., \& Artymowicz, P. 2001, ApJ, 557, 990

ten Brummelaar, T. A., et al. 2005, ApJ, 628, 453

Torres, G., Guenther, E. W., Marschall, L. A., Neuhäuser, R., Latham, D. W., \& Stefanik, R. P. 2003, AJ, 125, 825

Trilling, D. E., Koerner, D. W., Barnes, J. W., Ftaclas, C., \& Brown, R. H. 2001, ApJ, 552, L151

van Leeuwen, F. 2007, A\&A, 474, 653

Webb, R. A., Zuckerman, B., Platais, I., Patience, J., White, R. J., Schwartz, M. J., \& McCarthy, C. 1999, ApJ, 512, L63

Weinberger, A. J., et al. 2002, ApJ, 566, 409

Whitney, B. A., \& Hartmann, L. 1992, ApJ, 395, 529

Whitney, B. A., Kenyon, S. J., \& Gomez, M. 1997, ApJ, 485, 703

Wilner, D. J., Bourke, T. L., Wright, C. M., Jørgensen, J. K., van Dishoeck, E. F., \& Wong, T. 2003, ApJ, 596, 597

Wilner, D. J., D’Alessio, P., Calvet, N., Claussen, M. J., \& Hartmann, L. 2005, ApJ, 626, L109

Wilner, D. J., Ho, P. T. P., Kastner, J. H., \& Rodríguez, L. F. 2000, ApJ, 534, L101

Woillez, J., et al. 2010, Proc. SPIE, 7734, 30 